\section{$\underset{\substack{\text { hommes } \\ \text { \& migrations }}}{ }$}

\section{Hommes \& migrations}

Revue française de référence sur les dynamiques

migratoires

$1277 \mid 2009$

France - Allemagne

\title{
Revue de presse
}

\section{Mustapha Harzoune}

\section{OpenEdition \\ Journals}

\section{Édition électronique}

URL : http://journals.openedition.org/hommesmigrations/181

DOI : 10.4000/hommesmigrations. 181

ISSN : 2262-3353

\section{Éditeur}

Musée national de l'histoire de l'immigration

Édition imprimée

Date de publication : 1 janvier 2009

Pagination : $122-130$

ISSN : 1142-852X

Référence électronique

Mustapha Harzoune, "Revue de presse », Hommes \& migrations [En ligne], 1277 | 2009, mis en ligne le 29 mai 2013, consulté le 22 septembre 2020. URL : http://journals.openedition.org/

hommesmigrations/181; DOI : https://doi.org/10.4000/hommesmigrations.181

Ce document a été généré automatiquement le 22 septembre 2020.

Tous droits réservés 


\title{
Revue de presse
}

\author{
Mustapha Harzoune
}

\section{La Maison-Blanche sous le signe de la diversité}

1 Voilà c'est fait ! Les Américains ont élu Barack H. Obama à la Maison-Blanche. Après avoir installé aux commandes, de 1981 à 1989, Ronald Reagan, acteur de western puis, de 2001 à 2009, Georges W. Bush, texan aux allures de cow-boy évangéliste, voilà qu'ils placent à la tête du plus puissant pays de la planète, un jeune homme, cool, élégant, discret, à la tête bien faite et qui plus est Afro-américain. Barack H. Obama est "âgé de 47 ans, fils d'un père kenyan et d'une mère blanche américaine avec des racines à Hawaï et en Indonésie" (Los Angeles Times, 5 novembre 2008). Et le New York Times du même jour précise : "Un Américain, portant le nom de Barack Hussein Obama, le fils d'une femme blanche et d'un Noir qu'il connaissait à peine, élevé par ses grands-parents, loin de la puissance et de la richesse américaine, a été élu quarante-quatrième Président des États-Unis."

2 L'Europe, après s'être gaussée des Américains, ne tarit plus d'éloges. "Depuis mardi, ils sont nombreux à avoir perdu le droit de faire la leçon aux Américains" dit Jacques Julliard dans le Nouvel Observateur du 6 novembre 2008. Il ajoute tout de même : "Les Américains nous devaient bien ça: après huit années de George W. Bush, l'élection à la présidence des ÉtatsUnis d'un homme intelligent et généreux. Mais ce qu'ils ne devaient à personne, c'est la désignation d'un Noir, ou mieux peut-être, d'un métis pour le poste suprême."

3 Pour le Los Angeles Times, "L'antagonisme racial existe toujours. Mais, avec la victoire d'Obama, les électeurs ont montré que ces sentiments ne pèseraient plus du même poids sur la politique américaine." D'ailleurs, poursuit le quotidien de la côte Ouest, "l'ascension d'Obama symbolise la montée du multiculturalisme en Amérique" (5 novembre). 


\section{“En travaillant ensemble nous arriverons à panser nos vieilles blessures raciales" (Barack Obama)}

4 Dans un entretien mis en ligne le 5 novembre sur Bibliobs.fr, l'écrivain Alain Mabanckou reconnaît qu'en matière raciale, "un mur vient de tomber". Bernard-Henri Lévy, le 6 novembre dans Le Point, écrivait "une présidence Obama sera une date décisive, d'abord, dans l'histoire de ce que l'on persiste, aux États-Unis, à appeler la "question raciale" [...], même si, ajoutait-t-il, la montée en puissance du candidat "noir" a eu pour corollaire une remontée symétrique de toute une ultradroite nostalgique de la suprématie blanche et du bon temps ségrégationniste."

Claude Imbert, toujours dans Le Point, précise que "la fracture raciale, dixit Obama luimême, est loin d'être partout réduite. Ainsi l'élection affiche-t-elle moins le constat d'une éradication achevée du racisme que, chez les Blancs, un désir ineffable de rédemption pour un passé coupable."

6 Le même jour Judith Butler, professeure de rhétorique et de littérature comparée à l'Université de Berkeley Exubérance s'interrogeait sur le site Mediapart.fr: "Est-ce en dépit de sa race, ou en raison de sa race, qu'Obama a fini par devenir le représentant préféré de la nation ?" L'universitaire en appelait à un peu de "distance. [...] Peut-être est-il impossible d'éviter ce moment fantasmatique, mais soyons bien conscients qu'il est temporaire. S'il y a des racistes avérés pour dire "Je sais qu'il est musulman et terroriste, mais je voterai quand même pour lui", il y a sûrement des gens à gauche qui disent "je sais qu'il a abandonné les droits homosexuels et la Palestine, mais il est quand même notre rédemption"; et Judith Butler de poursuivre, Par quels moyens pourrons-nous préserver, tout en les masquant, de telles contradictions ? À quel prix politique ? [...] Si l'élection d'obama signifie que la majorité des électeurs sont prêts à être "représentés" par cet homme, alors cela redéfinit ce que "nous" veut dire: nous sommes une nation de races diverses, nous sommes racialement mixtes. Il nous offre l'occasion de reconnaître qui nous sommes devenus et qui nous devrions être, ce qui est une manière de résoudre la distance qui sépare la fonction représentative du Président de la foule des représentés. C'est un moment exaltant, sans aucun doute. Peut-il durer? Doit-il durer ?" Pour Albert Memmi, "L'élection d'Obama signifie que les Blancs seront maintenant plus aptes à accepter d'avoir un Noir à leur tête. C'était impensable avant" (Africultures.com, le 18 novembre 2008).

\section{Noir ? Métis ? Simplement américain !}

7 Pourtant, rappelle Alain Mabanckou le 7 novembre, sur le site Afrik.com, "Je n'ai toujours pas compris jusqu'à présent comment tout le monde le considère comme Noir alors qu'il est issu de l'union d'un père noir et d'une mère blanche. C'est aussi une forme de racisme venant des Noirs et des Blancs: tous les métis sont forcément des Noirs! Or Obama est le symbole de l'Amérique: un croisement de cultures et de races." Le 6 novembre, dans Le Nouvel Observateur, Jean Daniel ne disait pas autre chose: "Mais enfin, depuis le mardi 4 novembre à 19 heures, nous avons aux Etats-Unis, à la tête du pays le plus puissant du monde, un Président métis, issu de plusieurs civilisations, de plusieurs cultures. C'est-à-dire que la nation américaine accepte officiellement ce qu'elle était devenue et qu'elle se cachait à ellemême : la nation la plus multiculturelle, multiraciale et multimétissée." 
8 Etienne Dubuis, dans le quotidien suisse Le Temps du 7 novembre, montrait que les États-Unis étaient toujours une terre d'immigration et de plus en plus métissée : "Leur population s'accroît chaque année de 4,2 immigrants pour 1000 habitants, quand celle de l'Union européenne, soumise à un afflux historiquement élevé, augmente "seulement" de 2,4. Etre fils d'immigrant ou avoir vécu au loin, comme Obama, est des plus communs. Et ça le sera toujours plus : si, en 2005, 12 \% des Américains étaient nés à l'étranger, en 2050 ils seront $20 \%$ dans ce cas. "Quant à l'appartenance à une minorité ethnique ou raciale, elle est en voie de banalisation. Selon le Bureau américain du recensement, les "Blancs non hispaniques", qui constituaient encore $84 \%$ de la population en 1970, n'en représentent plus que $66 \%$.

9 Déjà minoritaires dans quatre États (Hawaï, la Californie, le Nouveau-Mexique et le Texas), ils le deviendront dans l'ensemble des États-Unis vers 2042, dans moins de deux générations. L'évolution s'annonce encore nettement plus rapide chez les enfants : tandis que $44 \%$ d'entre eux appartiennent aujourd'hui à une minorité, ils seront plus de $50 \%$ en 2023 et $62 \%$ en 2050.

10 "Le concept même de "groupe minoritaire" risque d'être bientôt périmé. Les membres de minorités étant de plus en plus nombreux et les mariages interethniques ou interraciaux se multipliant, il deviendra de plus en plus difficile de cataloguer les Américains en fonction d'un nombre raisonnable d'origines. Le recensement de 2000 a d'ailleurs reconnu cette évolution en permettant pour la première fois aux interrogés de se déclarer de deux races et plus.

11 Vous avez dit métis?"

\section{"Notre nation vaut plus que les parties qui la composent" (Barack Obama)}

12 Achille Mbembe, professeur d'histoire et de science politique à l'université du Witwatersrand (Johannesburg) en résidence aux États-Unis, voit en Obama "une certaine figure de notre monde" (Afrik.com, le 4 novembre). Mais une figure qui a dû prendre quelques distances. "À plusieurs égards, cet aggiornamento a nécessité une triple prise de distance : d'abord par rapport aux définitions canoniques de la condition noire aux États-Unis et aux modes d'action qui en ont historiquement découlé; ensuite par rapport aux stéréotypes qui ont historiquement permis de stigmatiser le signifiant noir et de "ghettoiser" la création politique afro-américaine. Cette prise de distance s'est également traduite par la mise entre parenthèses de l'Afrique dont il n'a pas prononcé le nom une seule fois au cours de cette campagne et qui, on le sait, a d'abord fait son apparition dans sa vie sous la figure du "père absent", mais dont, conséquence de la malédiction généalogique, on ne peut se défaire. L'écart s'applique également à son propre prénom "Hussein", à consonance musulmane, dans ce pays où beaucoup sont persuadés que l'Islam est une religion suicidaire. Grâce à cette triple excision, Obama n'est jamais apparu aux yeux de la majorité de l'électorat comme "le candidat noir". D'ailleurs, écrit BHL dans Le Point du 6 novembre, "Il faut l'écouter, quand, dans chacun de ses discours, il cite le "E pluribus unum" de Virgile d'où la devise du pays est tirée et qu'il traduit par "Notre nation vaut plus que les parties qui la composent." Et il faut mesurer le tabou qu'il brise, enfin, quand il s'adresse aux Noirs eux-mêmes pour les inviter à ne plus imputer au seul racisme la source de leurs maux. Là aussi, c'est un propos nouveau. C'est le message du dernier King, celui des années 1967-1968; mais c'est un propos nouveau par rapport à tous les discours faisant de l'appartenance à la "race" le dernier mot de l'identité de chacunet son impasse." 


\section{Le plus compétent, le plus rapide et la plus belle}

13 Alors qu'un vent d'obamania balaye l'Hexagone, “Pierre N'Gahane, 45 ans, né au Cameroun, a été nommé, mercredi [12 novembre] en conseil des ministres, préfet du département des Alpes-de-Haute-Provence." Pour Pierre N'Gahane, sa nomination comme "premier préfet noir d'origine africaine de France" tient davantage d'un effet de calendrier que d'un effet Obama (http://www.bbc.co.uk/french/news/story/ 2008/11/081112_france_blackprefet.shtml).

plus tard, M. Nacer Meddah est nommé, lui, préfet de la Seine-Saint-Denis. Dès le lendemain, l'Union des associations musulmanes de la Seine-Saint-Denis (UAM 93) s'est félicitée, sans doute par goût du métissage et de la laïcité, de cette nomination qu'elle interprète comme "un geste fort à destination des musulmans de France" (dépêche AFP du 11 novembre).

15 Suite à cette déclaration, Le Parisien du 12 novembre écrit que "Nacer Meddah tient [...] à préciser qu'il n'est pas musulman et revendique sa laïcité. [...] Je veux chasser les stéréotypes et les idées reçues sur la Seine-Saint-Denis. Il y a des difficultés ici, comme il en existe ailleurs et partout", ajoute celui qui veut "se plonger dans les réalités locales avant de se prononcer plus avant sur ses priorités."

16 Que Pierre N'Gahane et Nacer Meddah se rassurent: ils ne sont pas les seuls à faire l'actualité du métissage. Ils sont même en bonne compagnie : Miss France 2009 est ellemême une Franco-américaine.

17 Le 8 décembre, l'AFP rapportait :

18 "en couronnant pour 2009 l'étudiante métisse franco-américaine Chloé Mortaud, le public et le jury Miss France ont élu pour la première fois une candidate possédant une double nationalité." Et de préciser :

19 "La jeune fille de 19 ans aux yeux noisette et aux cheveux châtain foncé, qui mesure 1,80 mètre, a une mère originaire de l'État du Mississipi arrivée en France il y a 25 ans."

20 Autre succès d'un métis, le 2 novembre au Japon, le Britannique Lewis Hamilton remportait le Championnat $\mathrm{du}$ monde de Formule 1. "Hamilton, la jolie face du multiculturalisme", titrait un article du Guardian repris par Courrier international du 3 novembre. On y apprend que "ses attachés de presse ont interdit aux journalistes de lui poser des questions d'ordre racial ou ethnique". "Qu'il ait surgi à un moment aussi difficile pour l'identité nationale et raciale du pays est plus qu'intéressant. Dans des périodes comme celle que nous vivons, quelqu'un comme Hamilton peut devenir la face acceptable du multiculturalisme britannique, estime Carrington [professeur à l'université du Texas à Austin]. Il est originaire de Stevenage et non de Moss Side (quartier sensible de Manchester) ou de Chapelton. Il est métis. Il est affable et modeste. Il vient d'une famille stable et il est proche de son père. Il représente la différence sans être trop différent."

21 Il serait donc, lui, présentable... Comme Obama. Le 10 janvier dernier sur son blog le toujours vert Walter Lewino (84 ans) résume : “Qu'est-ce qu'on en a foutre de la couleur de sa peau, c'est un homme comme les autres, sans doute ni mieux ni pire, et c'est en tant qu'homme qu'il le faut apprécier et juger. Finalement l'obamania ne serait-elle pas la forme la plus sournoise du racisme. Il est beau, il est bon, il est gentil... parce qu'il est Noir."

22 Alors basta! Barack Obama est le plus compétent, Lewis Hamilton, le plus rapide et Chloé Mortaud, la plus belle! Et si Yannick Noah, Danny Boon et Zinedine Zidane 
restent, eux, les chouchous des Français en 2008, ce n'est pas parce que l'un est d'origine camerounaise et les deux autres d'origine kabyle.

\section{Statistiques ethniques !}

Mais enfin, forts d'un voyage d'étude sur la diversité aux États-Unis organisé par l'Institut Montaigne, Pascal de Jenlis, président-fondateur du groupe Hameur et Philippe Manière, directeur général dudit Institut, affirmaient sur le site de La Tribune, le 12 novembre : "La première étape, fondatrice, d'un vrai changement, serait d'admettre enfin la légitimité d'un décompte. Dans l'administration, dans l'entreprise, dans l'armée, et en particulier au plus haut niveau, quel est le pourcentage de personnes issues de l'immigration et des minorités visibles?"

Faut-il s'étonner que les conclusions de ce "think tank d'inspiration libérale" (L'Express du 3 septembre), soient partagées par quelques minorités qui profitent de l'obamania pour rappeler leurs revendications? Ainsi selon LeMonde.fr du 10 novembre, le Conseil représentatif des associations noires (Cran) "veut que la République s'applique à tous". Après avoir été reçu le 10 novembre à l'Élysée par Cédric Goubet, le chef de cabinet de Nicolas Sarkozy, "Patrick Lozès, président du Cran, estime avoir été en partie entendu, notamment sur l'éventuelle approbation de statistiques ethniques. Un outil qu'il juge nécessaire pour évaluer la discrimination [...]. Il estime également avoir reçu une réponse encourageante sur "l'action positive", une idée qu'il défend depuis la création du Cran en 2005, mais qu'il distingue de la discrimination positive."

\section{La montagne Obama accoucherait-elle de la souris Hortefeux?}

"Evoquant la "leçon" que constitue pour la France la victoire de Barack Obama à la présidentielle américaine" (LeMonde.fr, 9 novembre), l'industriel et président de l'Agence nationale de rénovation urbaine (Anru), Yazid Sabeg publie un "Manifeste pour l'égalité réelle", dans le Journal du Dimanche du 9 novembre. Au programme: "Engager des politiques publiques qui combattent les conséquences sociales des discriminations, systématiser les politiques volontaristes de réussite éducative et la promotion des talents dans les quartiers populaires, promouvoir des politiques urbaines qui permettent de réaliser la diversité sociale et de peuplement, inciter fortement les employeurs et le premier d'entre eux, l'État, à mettre en place des politiques de promotion de la diversité fondées sur l'obligation de résultat, limiter les mandats électoraux pour forcer le renouvellement du monde politique, soumettre les partis politiques à un pacte national de la diversité et organiser un Grenelle de l'égalité réelle et de la diversité."

Le 12 novembre sur le site du Figaro, on apprenait que le Manifeste "soutenu par JeanFrançois Copé, Dominique Voynet, Arnaud Montebourg, Carla Bruni-Sarkozy ou encore Patrick Devedjian", est aussi du goût de Brice Hortefeux. "J'approuve totalement. Cette initiative souligne deux réalités. Les politiques d'intégration ont globalement échoué. La raison, c'est qu'il n'y a pas vraiment eu de politique d'immigration depuis une trentaine d'années. Il faut que les deux plateaux de la balance soient à l'équilibre": la maîtrise des flux migratoires et l'intégration. Et d'ajouter : "il n'y a pas de fatalité" pour "les immigrés légaux [...] respectant les lois qui sont les nôtres". 
L'intégration de ces "immigrés légaux" en passerait par "la connaissance de la langue avant d'arriver sur le territoire, de nos valeurs, de nos institutions". Quant à la Marseillaise, "ce n'est pas une mélodie, ce doit être une pédagogie". "Aujourd'hui, un immigré sur trois ne parle pas notre langue. Comment voulez qu'il trouve un emploi, fasse ses courses, comprenne le cursus scolaire ?" "Brice Hortefeux insiste enfin sur les valeurs, notamment l'égalité hommes-femmes." La montagne Obama accoucherait-elle de la souris Hortefeux?

\section{La danse de Saint-Guy de la "diversité"}

Le 17 décembre, à l'occasion de son discours sur la diversité, Nicolas Sarkozy dresse le constat d'un "décalage toujours plus grand qui saute aux yeux entre la diversité de la société française et l'homogénéité sociale et culturelle toujours plus grande des élites que produit notre système éducatif". Il fait de "l'égalité des chances, [...] la priorité". Si "l'objectif" est de "relever le défi du métissage", pour autant, "c'est par le critère social qu'il faut prendre le problème parce que les inégalités sociales englobent toutes les autres". Il demande à son gouvernement de remettre "un plan d'action d'ici le mois de mars". À cette occasion, Yazid Sabeg est "désigné commissaire à la Diversité et à l'Égalité des chances". Les orientations avancées lors de ce discours portent notamment sur : la nécessité de recourir à des "outils statistiques" ; à la suppression progressive de la carte scolaire ; à la fermeture de certaines écoles pour "mieux assurer le brassage social"; et à la mise en œuvre rapide du plan "Espoir banlieue". "Les minorités visibles de l'UMP, réunies au sein du CDR, le Cercle de la diversité républicaine" seront sans doute satisfaites, elles qui "veulent secouer leur parti et le gouvernement pour qu'enfin se concrétise une France métissée". Le 4 décembre, elles ont présenté une douzaine d'“actions positives d'intégration" dont elles réclament une "mise en ceuvre rapide" (Le Monde, 5 décembre). Et le CDR - comme Yazid Sabeg, l'Institut Montaigne et le Cran... - y va de son programme et de ses propositions : il "demande àce que soit enfin publié le décret d'application $d u C V$ anonyme, rendu obligatoire par la loi sur l'égalité des chances de 2006, mais jusqu'ici resté lettre morte. Il propose que l'attribution des marchés publics soit conditionnée à une représentation des minorités dans les conseils d'administrations des entreprises retenues. Dans la fonction publique, le CDR prône une diversification des voies de recrutement et dès lors, une généralisation des systèmes de type troisième voie d'accès." Et comme tous trinquent au même élixir, "les minorités visibles de l'UMP réclament la création d'un ministère de la Diversité, doté d'un budget propre et d'une administration et elles relancent l'idée d'instaurer des statistiques de la diversité." Sans oublier (c'est le nouveau commissaire à la Diversité qui va être content), “ l'organisation en mars 2009 d'un Grenelle de la diversité."

\section{"Diversité : l'échec de Nicolas Sarkozy ?" (Sylvie Pierre-Brossolette)}

Yazid Sabeg aura tout de même du pain sur la planche à lire les propos du président de l'Olympique de Marseille, Pape Diouf. "Cet ancien journaliste et agent de joueurs est le seul Noir à occuper un tel poste dans le football européen. [...] Comme joueurs, les Afro-Arabes sont très présents sur les terrains, mais dès qu'il faut mettre en place des équipes dirigeantes, ils disparaissent. C'est profondément choquant! Je suis une anomalie car, avant moi, il n'y a pas d'exemple. Je suis devenu symbolique, car j'occupe un poste qu'on ne donne pas aux Noirs et aux Arabes. [...] Voyez-vous des Noirs ou des Arabes, hormis sous forme de saupoudrage, être des 
ministres d'envergure? En connaissez-vous qui soient des leaders au sein des partis politiques? Patrons d'une grande entreprise nationalisée ? Il y en a forcément qui sont en position d'occuper ces postes-là. L'égalité sociale ethnique interviendra le jour où on nommera à des postes importants un Arabe ou un Noir incompétent. Les féministes disaient cela sur l'égalité des sexes. Nous n'en sommes pas là" (Le Monde du 22 décembre 2008). Alors que Yazid Sabeg entre au gouvernement, le Haut Conseil à l'intégration voit s'éloigner un peu de ce "Tout-Monde" théorisé par Édouard Glissant, avec la démission, début décembre, de Jacky Dahomay (suivie le 16 décembre de celle d'Édouard Glissant) pour qui il serait bon "aussi [de] réviser cette identité républicaine hypocrite qui a du mal à s'ouvrir à la diversité. Et quand on constate que monsieur Brice Hortefeux, ministre de cet affreux ministère de l'Intégration, de l'Identité nationale et de l'Immigration, aux relents franchement vichyssois, se permet de réunir, à Vichy précisément, les ministres européens chargés des questions d'immigration, on peut légitimement penser qu'il y a là une continuité conservatrice inquiétante" (rue89.com, le 10 décembre). Et Sylvie Pierre-Brossolette qui, le même jour, sur le site du Point, affirme sans barguigner : "Diversité: l'échec de Nicolas Sarkozy"! Pour la journaliste, "L'opération "diversité" de Nicolas Sarkozy, qui contribua beaucoup à son état de grâce à la suite de l'élection présidentielle, se poursuit dans la confusion, voire dans l'échec." Et de biffer d'un trait de plume "la garde des Sceaux [qui] n'a eu ni la manière ni la légitimité pour s'imposer [...]. La voilà devenue un boulet pour le régime, un crève-cœur pour les tenants de l'intégration." Même régime de faveur pour Rama Yade qui aurait "déçu" M. le Président.

"Passons, écrit la journaliste, sur le cas de Fadela Amara, sympathique à une grande majorité de Français, mais qui n'a pas su arracher un véritable plan Marshall pour les banlieues." Restons, au contraire, avec Fadela Amara. Le 2 novembre, elle demandait dans les colonnes du JDD que les formations politiques "aillent chercher des candidats issus de l'immigration, de toutes les origines et qu'ils les offrent aux électeurs. Les gens sont prêts, c'est le système politique qui est en retard. [...] le travail ne se fait pas assez vite en haut." Et voilà que, le 22 décembre dans l'émission politique du dimanche soir sur France Inter, à la question: "Ne regrettez-vous pas que le gouvernement renonce à faire de la discrimination positive pour promouvoir la diversité ?", elle répond: "Pas du tout. Je fais cinq visites de terrain par semaine dans les quartiers. Leur demande est très claire: ils veulent être traités comme tout le monde. Ça s'appelle l'égalité des chances."

Concernant les lenteurs du plan "Espoir banlieues", elle déclare : "Vous savez, dans ce pays, une partie de l'élite ne souhaite pas voir émerger les enfants d'ouvriers. Ça commençait à m'agacer sérieusement de voir qu'en haut lieu certains énarques freinaient des quatre fers. J'ai tapé du poing sur la table en le disant au président de la République, avant son discours sur la diversité."

\section{“E pluribus unum" (Virgile) : la société montre l'exemple}

32 Au cœur de cette agitation médiatico-politicienne, une question circule : "Barack Obama aurait-il pu être élu en France ?" Comme on a pu le constater, chacun a une solution à la bouche et son bréviaire en poche. Philippe Bernard sifflait la fin de la récréation dans Le Monde dès le 20 novembre avec son article sur : "les pièges de l'obamania à la française". Dans une "France monocolore" à "l'homogénéité stérilisante", il fustigeait - citant Christine Lagarde ou Carla Bruni-Sarkozy - "l'extrême confusion", la "récupération politique" et la "manipulation" en vogue. 
Pour Philippe Bernard, "Les Américains ont choisi le candidat le plus talentueux et le plus crédible en dépit, et non à cause, de la couleur de sa peau. [...] Il apparaît d'ailleurs pour le moins paradoxal que la France rouvre le débat sur l'accès aux responsabilités des personnes issues de l'immigration au moment même où le nouveau Président américain privilégie les facteurs sociaux sur les variables raciales dans son analyse des fractures de la société américaine."

4 Après avoir montré "à quel point la République a failli à tenir sa promesse d'égalité" il détaille ces "réformes engagées [qui] pèsent contre la diversité prétendument célébrée : la diminution des exigences légales en matière de mixité sociale dans l'habitat, la suppression de la carte scolaire, le refus de généraliser par la loi à toutes les formations supérieures sélectives le quota de $10 \%$ d'étudiants venant de tous les lycées du pays, outremer compris, et l'asphyxie de la rénovation urbaine. Au surplus, le climat de suspicion à l'égard des étrangers, et en particulier des couples mixtes qui prévaut en France rendrait aléatoire le mariage des parents d'un futur Obama français. [...] À l'heure où un métis accède au bureau Ovale et où Les Verts allemands choisissent un fils de Turc pour leader, écrit Philippe Bernard, la diversité en France irrigue et enrichit déjà les ateliers, les commerces, les salles de professeurs et les prétoires. À peu près tous les milieux, à l'exception notable de la haute hiérarchie des partis politiques."

5 Philippe Bernard aurait pu ajouter à sa liste... les alcôves. Dans le magazine Elle, du 8 décembre 2008, le sociologue Gérard Neyrand indique que "la société française est plus libérale et plus ouverte que par le passé. Elle accepte de mieux en mieux les couples mixtes, instrument d'intégration par excellence. Paradoxalement, l'arsenal législatif agit dans le sens contraire et on assiste depuis plusieurs années à un regain de suspicion à l'égard des couples binationaux, soupçonnés de mariages blancs. [...] Ce durcissement politique est d'ailleurs à l'origine de la création, en juin 2007, de l'association des Amoureux au ban public, qui défend le droit à s'aimer des conjoints de nationalités différentes."

Selon le magazine, il y aurait en France, "plus de 39000 mariages franco-étrangers par an. Un chiffre qui a presque doublé en dix ans, représentant aujourd'hui près de $14 \%$ des passages devant le maire. Ce qui fait de l'Hexagone un champion européen en la matière." Et de citer le philosophe Vincent Cespedes : "La mixité amoureuse, c'est l'avenir de l'humanité. Pour trois raisons : le bilinguisme est un enrichissement prodigieux, car la langue est une façon de voir le monde. Plus on en connait, plus on voit la réalité selon des prismes différents. Ensuite, on sait que le brassage, le mélange des gènes est une vraie richesse. Il n'y a qu'à voir les ravages de la consanguinité. Enfin, quand la mixité est incarnée dans un être, ce mélange implique forcément une plus grande tolérance, ça ouvre les points de vue." "Les couples mixtes, c'est l'universalisme mis en pratique. Et, sur ce point, la société a pris de l'avance sur les politiques." 